\title{
Civilisations
}

Revue internationale d'anthropologie et de sciences

humaines

$41 \mid 1993$

Mélanges Pierre Salmon II

\section{Intentions missionnaires et perception africaine : quelques données camerounaises}

\author{
Philippe Laburthe-Tolra
}

\section{OpenEdition}

\section{Journals}

Édition électronique

URL : http://journals.openedition.org/civilisations/1708

DOI : 10.4000/civilisations. 1708

ISSN : 2032-0442

\section{Éditeur}

Institut de sociologie de l'Université Libre de Bruxelles

\section{Édition imprimée}

Date de publication : 1 septembre 1993

Pagination : 239-255

ISBN : 2-87263-094-5

ISSN : 0009-8140

Référence électronique

Philippe Laburthe-Tolra, «Intentions missionnaires et perception africaine : quelques données camerounaises », Civilisations [En ligne], 41 | 1993, mis en ligne le 29 juillet 2009, consulté le 19 avri 2019. URL : http://journals.openedition.org/civilisations/1708; DOI : 10.4000/civilisations. 1708

Ce document a été généré automatiquement le 19 avril 2019

(c) Tous droits réservés 


\title{
Intentions missionnaires et perception africaine : quelques données camerounaises
}

\author{
Philippe Laburthe-Tolra
}

\section{NOTE DE L'AUTEUR}

De rencontres trop brèves, mais riches, avec Pierre Salmon, j'ai gardé en mémoire son encouragement amical à étudier l'histoire des variations de mentalités. Celle-ci se constate en particulier dans l'événement, à la réflexion bien étrange, que constitue la tentative de conversion religieuse, c'est-à-dire l'irruption d'un état d'esprit et d'une volonté de changement forgés ailleurs dans une société qui jusque là vivait autrement. On voudra bien trouver ici, en hommage au cher professeur Salmon, quelques faits concernant la rencontre des missionnaires catholiques avec les Beti du Cameroun méridional, éléments que j'espère développer dans un ouvrage ultérieur, en montrant qu'ils entraîneront un changement effectif dans la manière de voir des Beti, mais ausi dans celle des missionnaires.

1 Le 14 juillet 1884 fut célébré comme une fête, dans l'estuaire du Wouri, l'annexion du Cameron à l'Empire allemand, annexion paradoxale, puisqu'elle résultait d'un vieux désir des rois duala et d'une décision prise à contre-coeur par Bismark. Mais ceci est une autre histoire.

2 A l'époque il n'y avait sur place, depuis 40 ans, que des missionnaires baptistes. Les catholiques cherchèrent à se faire admettre à leur tour, et y parvinrent non sans peine en 1890.

3 La langue de bois anti-colonialiste fait des missionnaires les complices de l'entreprise coloniale (Cf. Mohr 1965, Messina 1988). Complices "objectifs", certes. Napoléon disait déjà à leur propos : 
"Je les enverrai prendre des renseignements sur l'état des pays. Leur rôle les protège et sert à couvrir des desseins politiques et commerciaux" (in Dansette 1965 : 150).

4 Mais c'est méconnaître que le christianisme, même comme religion d'Etat, a presque toujours constitué un contre-pouvoir pour l'autorité politique, marquant par exemple les limites de l'absolutisme royal et de l'arbitraire seigneurial. L'un des attraits du protestantisme pour les princes fut l'élimination de l'appel extra-national au Pape.

Les colonisateurs allemands n'ignoraient pas l'histoire. Bismarck craignait en outre l'effet désastreux outre-mer des rivalités confessionnelles. Il fit donc tout pour empêcher les Missions de prendre pied dans ses protectorats (Berger 1978 : 25). Il remplaça les pasteurs anglais déjà présents au Cameroun par les germanophones de la Mission de Bâle, qu'il espérait plus soumis, et il louvoya avec cynisme pour éviter l'arrivée des catholiques (op.cit. : 30-32), qui dut être imposée par le Reichstag grâce à Windthorst et au Zentrum.

6 Agnostiques pour la plupart, les officiers et fonctionnaires allemands partageaient les idées de Voltaire et de Napoléon sur la nécessité didactique de la religion. L'empereur n'était-il pas chrétien? A l'école gouvernementale de Douala, le premier instituteur d'Etat (Reischsschulinspektor) fut Christaller, né en Gold Coast d'un pasteur de la mission de Bâle. Christianisme et cantiques étaient conçus comme partie intégrante de la culture à diffuser.

7 L'indépendance chrétienne des protestants n'en irritait pas moins le gouvernement colonial. Il semble bien que le chancelier Leist ait tenté de faire tuer ce premier instituteur officiel qui enseignait en duala, nommait son fils Ndumbe en l'honneur du roi Bell, et qui constituait certes un témoin gênant quand les mercenaires dahoméens du gouvernement s'insurgèrent, en décembre 1893, par suite des abus lubriques et sadiques de Leist sur leurs femmes.

Christaller se réfugie chez le pasteur de Bonabéri, ou le roi Bell en personne vient le prévenir qu'il n'a rien à craindre.

Mais Leist, gouverneur par intérim, intime l'ordre à l'instituteur de se réfugier sur le bateau "Nachtigal". Avec sa femme et son bébé, il est contraint de s'embarquer sur une chaloupe ; "laquelle, à leur grande-surprise, les amena non au "Nachtigal", mais au "Soden", qui commença aussitôt à tirer de ses deux mitrailleuses sur l'endroit de l'émeute. Etait-ce par erreur ou de propos délibéré qu'on les amenait en plein combat? Le chancelier voulait-il ainsi se venger du remuant instituteur et de la mission évangélique qu'il haïssait à mort? Aurait-il vu d'un assez bon oeil qu'une balle des insurgés atteignît Christaller? En tout état de cause, c'était une façon très étrange de secourir les gens" (Boeckeler 1897 : 131).

Le petit Ndumbe hurle à chaque salve ; sa mère pense qu'elle aimerait mieux être tuée vite plutôt que de devenir folle dans cet enfer. Pendant ce temps, le chancelier et les autres blancs résident tranquillement à bord du "Nachtigal" qui se réfugie au loin (ils vont y rester 9 jours). Durant les pauses, Christaller demande par trois fois à ce qu'au moins sa femme et son fils aillent à bord du "Cyclop" qui sert de navire hôpital à l'écart. Point de réponse. On les débarque dès la fin de l'émeute, et ils retrouvent leur école criblée de balles allemandes.

(Tous les rebelles hommes attrapés alors seront pendus le $1^{\mathrm{er}}$ janvier 1894, les femmes emprisonnées ; d'autres hommes pris par la suite seront "grâciés" pour des travaux forcés à perpétuité. Mais l'affaire fait scandale au Reichstag. Leist traduit devant un conseil de discipline, sanctionné et révoqué, devra s'exiler à Chicago).

8 C'est dire que les rapports entre catholiques et officiels seront a fortiori difficiles. Donnons-en quelques traits. 
9 Un premier contingent de missionnaires catholiques débarque le 25 octobre 1890, comprenant deux prêtres seulement, cinq frères coadjuteurs et un scolastique (séminariste religieux). Dès sa première visite, le supérieur, le P. Vieter, se trouve en butte à une attaque de Puttkamer, alors gouverneur par intérim. Comme le Préfet apostolique se réfère aux décisions du Parlement, il s'entend répliquer : "Ici, c'est moi qui commande et non le Reichstag !".

10 Vieter rapporta cet incident à Rome, dans une lettre confidentielle, mais que ses supérieurs publièrent. Puttkamer fut blâmé (cf. Tabi 1971 : 22).

11 Par la suite, et malgré les efforts diplomatiques de Vieter, les sujets de tension ne manquèrent pas. Les principaux furent les exactions dont les Blancs se rendaient coupables, et leur exploitation des Noirs dans le système des grandes plantations. A cause de sa défense inconditionnelle des Camerounais, le P. König, qui publiait des articles vengeurs dans les journaux allemands, fut condamné à cinq jours de prison ferme (cf. Criaud-Vieter $1989: 46,78,83$ ).

12 Autre élément de discorde : la discrimination raciale jusque dans la mort. Douala avait son cimetière "blanc". Querelle pour parvenir à acheter une terre où enterrer tous les catholiques, Blancs et Noirs, ensemble (Skolaster 1924 : 114).

13 Un autre sujet de discussion fut l'acquisition des terrains nécessaires aux missions. Il fallut les payer plusieurs fois : une première fois au chef qui le cédait, une seconde ou même une troisième fois à l'administration qui, par fiction juridique, en 1896, avait déclaré toute terre vacante propriété de la Couronne.

Ainsi à Engelberg : le contrat de cession avec le chef Efesoa, propriétaire coutumier, se voyant refusé toute valeur juridique, Vieter achète le terrain pour 250 marks au gouvernement, et paye à cette occasion 1.000 marks à l'arpenteur. Mais en 1897 Puttkamer veut tout reprendre parce que le terrain n'est pas assez cultivé, donc "vacant" ! -A Victoria, Vieter a acheté une maison 1.000 marks sur un terrain dont Puttkamer veut bien lui faire cadeau, mais en exigeant qu'il renonce à s'établir à Buéa où les deux collines que le chef Kuba a promises à la mission par écrit sont voulues par le gouvernement (Criaud-Vieter 1989 : 77).

14 Nouveau conflit lorsque l'administration, au mépris du Traité de protectorat, entreprit en 1913 d'exproprier les rois duala et leur peuple du secteur qu'ils occupaient en bordure du Wouri, pour le réserver aux Européens.

"Les Noirs se défendent de toutes leurs forces et envoient des télégrammes au Reichstag, etc... mais tout cela sera inutile", regrette Mgr Vieter (op.cit. : 169). "La force prime le droit et la force est le droit! Ce sont là des principes contre lesquels le Noir ne peut rien (...). H est évident que cela ne plaît pas à la Mission (...). Tous les Européens, excepté le commerçant Steier, sont contre ces mesures. Le chef de circonscription Jacob a sur sa demande obtenu une affectation de Douala à Kribi parce qu'il ne veut pas participer à ce forfait culturel".

$\mathrm{M}^{\mathrm{gr}}$ Vieter écrit alors au prince Alois von Löwenstein, protecteur de la mission, pour lui demander d'intercéder au Reichstag et auprès des Affaires Etrangères. En vain. Les Douala passèrent dans une dissidence qui aboutit à la pendaison du roi Rudolf Duala Manga Bell le 8 août 1914.

Quels sont les hommes qui s'opposent ainsi au pouvoir politique?

La congrégation catholique qui se présente au Cameroun est à l'époque allemande (1890-1915) la "Pieuse Société des Missions" (P.S.M.) qui a depuis changé son nom en S.A.C. ("Société d'Action Catholique"). Fondée à Rome en 1835 par S. Vincent Pallotti, elle 
eut d'abord pour objectif l'apostolat intellectuel, mais non sans désir d'actions lointaines. La mission du Cameroun lui offrait par ricochet l'occasion d'essaimer en Allemagne.

En 1916, elle sera remplacée par les Pères français de la congrégation du Saint Esprit, avec la même ambition, inspirée ici par le P. Libermann, de reconstituer outre-mer la "chrétienté" qui est en train de se dissoudre en Europe.

Max Weber rappelle dans l'Ethique protestante comment, en réaction contre le laxisme et l'hypocrisie reprochés à Rome, certains courants calvinistes ou puritains avaient entrepris de transformer le monde en un vaste couvent. La Contre-Réforme catholique ne sera pas en reste : elle tente d'organiser la vie du chrétien ordinaire selon des habitudes méticuleuses en harmonie avec les principes évangéliques. Certains préceptes doivent informer la vie de tous les fidèles: telles les prières du matin et du soir, si possible en famille, le bénédicité au début et les grâces à la fin des repas, l'angelus trois fois par jour, sans parler bien sûr d'obligations strictes comme l'abstinence de viande à certains jours, la messe dominicale, le carême, la fréquentation des sacrements, auxquelles s'adjoignent de nombreuses dévotions facultatives mais très pratiquées comme les vêpres et saluts, la récitation du chapelet, les processions et pélerinages, ainsi que l'habitude des "petits sacrifices" offerts par amour de Dieu pour le salut des âmes.

Les régions ferventes de l'Europe en seront marquées durant trois siècles, en particulier les milieux ruraux dont la plupart de nos missionnaires au Cameroun sont issus : souvent Westphaliens, Alsaciens, Bretons.

21 Vers la fin du XIX ${ }^{e}$ siècle, malgré les changements (ou peut-être à cause d'eux), cette paysannerie s'ancre dans son catholicisme autour de la paroisse et de l'école religieuse, avec des habitudes qui vont d'une pudeur corporelle scrupuleuse aux lectures édifiantes de la "bonne presse" (même dans des familles très pauvres). Suhaud (1976: 90), pour les années 1920-30 (et a fortiori pour les années 1880 ou 1900), reconnait que ces habitudes rigoureuses sont en cohérence avec celles qui, en cas de "vocation", attendent au petit séminaire ces enfants de condition modeste, auxquels s'adjoignent parfois quelques petits bourgeois, fils d'artisans, de commerçants, exceptionnellement de notables.

Le futur $\mathrm{M}^{\mathrm{gr}}$ Hennemann, successeur de Mgr Vieter, et deuxième Vicaire apostolique du Cameroun, raconte sa vocation au chapitre I de son livre de souvenirs: Trajet et Travaux d'un missionnaire d'Afrique. Orphelin à dix ans, il est recueilli par un oncle ami des missions qui lui fait distribuer des images de propagande, des médailles et des bulletins pour soutenir les Pallotins : "Les pauvres Africains ont besoin d'amis". Le petit Franz souhaite en être et son oncle l'inscrit omme "tertiaire pallotin". Quand l'Etoile d'Afrique se met à paraître, en 1824, c'est lui qui en assure la diffusion au village (à Holthausen en Sauerland). "Deux marks par an pour ces petits cahiers de 4 pages, c'est trop, tu n'auras pas beaucoup de clients" soupire son tuteur. Le garçon en trouve cependant, tout en dévorant avec enthousiasme la littérature qu'il colporte. Il calcule qu'un missionnaire meurt automatiquement au bout de deux ans de séjour en Afrique: cet héroïsme le fascine. Après des études secondaires brillantes et mille projets, voici que l'un de ses amis entre au séminaire des Pallotins. Il le suit, ne réalisant son changement de vie qu'une fois franchi le seuil du couvent. Les occupations au séminaire lui font paraître les jours trop courts, du travail des champs et du lavage des dortoirs aux leçons d'anglais, de philosophie et de théologie. Les départs en mission étaient l'occasion d'émouvantes cérémonies. Seules les excursions lui paraissaient trop longues, mais elles constituaient un entraînement qu'il ne regrettera plus au Cameroun. Ordonné le 29 juin 1907, il s'embarque malgré une bronchite sur le Lucie Woermann pour atteindre Douala après trois semaines de traversée. A terre, il ingurgite de bon appétit les mets africains: mouton poivré, macabo, plantains, 
sauce à l'huile de palme, vin de raphia. "Avez-vous un bon estomac ? lui demande l'évêque. - De Westphalien, Monseigneur! - Alors, vous êtes fait pour Yaoundé, c'est là que je vous envoie !" (N.B. M ${ }^{\text {gr }}$ Vieter venait aussi de Westphalie).

Nombre de ces paysans ou artisans qui n'auront pas su ou pu partir au petit séminaire formeront une cohorte importante de religieux laïcs, les "Frères" coadjuteurs, auxiliaires dont le rôle en mission, ainsi que celui des religieuses, sera fondamental à la fois à titre de techniciens manuels ou professionnels, et d'intermédiaires, de cadres moyens pourraiton dire, entre le "père" prêtre et le peuple autochtone.

Les garçons destinés au sacerdoce vont subir une longue formation en internat : six à sept ans d'études secondaires au petit séminaire, ou à l'école apostolique, un ou deux ans de noviciat et de juvénat, cinq à six ans de grand séminaire ou scolasticat, où ils vont se former à la vie et à la spiritualité de leur congrégation, à la philosophie thomiste, aux différents domaines de la théologie (dogmatique, morale, patristique, etc.), avant de prononcer les voeux définitifs de pauvreté, de chasteté et d'obéissance, de recevoir le sacrement de l'Ordre et d'être envoyés en mission.

Tout au long de cette formation se pose le problème de l'authenticité de la vocation présumée, afin d'écarter les candidats douteux, ou inaptes à tenir les divers engagements qu'ils auront à prendre pour le reste de l'exitence.

L'aspect ascétique de cette formation, dépouillement de soi, mortification, humilité, vie communautaire, remonte droit aux Pères du Désert, Antoine, Pacôme, au IV ${ }^{\mathrm{e}}$ siècle (voire au-delà, aux Esséniens, etc.).

6 La mystique et la pastorale varient davantage suivant les congrégations; elles sont généralement marquées par l'esprit propre du fondateur.

Les études restent très marquées des avanies subies par l'Eglise depuis l'irruption du libre examen et la montée de l'esprit critique dans tous les domaines. Dans l'ensemble, depuis le Siècle des Lumières, une certaine routine de la pensée cléricale s'est instaurée, que le renouveau interne provenant de la vraie philosophie thomiste ou de l'exégèse biblique n'ébranle pas avant la Première Guerre Mondiale (cf. Dansette 1965).

L'origine puritaine et modeste des missionnaires les pousse d'ailleurs à se méfier de leurs propres études et à préférer la rigidité à la souplesse : le P. Stoll s'étonnera qu'après avoir reçu au scolasticat la doctrine morale probabiliste (c'est-à-dire ouverte, compréhensive) de $\mathrm{S}$. Alphonse de Ligori, ses confrères se montrent si rigoristes vis-à-vis d'Africains qui auraient au contraire requis de l'indulgence. C'est que tous restent marqués par un milieu qui prônait comme des fétiches le travail, l'épargne, la pureté des moeurs, vertus qu'ils s'indignent de trouver si étrangères aux "paysans" africains, dont ils auraient voulu faire les homologues de leurs propres parents.

Cette formation en reste à une vue nécessairement simplifiée du christianisme, analysé selon le catéchisme en vérités à croire et commandements à observer. Ceux qui y adhèrent seront sauvés, ceux qui s'en écartent sont perdus. L'apostolat est conçu comme un duel à mort entre bien et mal, lumière et ténèbres; ce qui ne favorise certes pas la compréhension de la société "païenne".

Mais pourrait-il en être autrement pour qui va vraiment sacrifier sa vie à la cause de la conversion? On ne peut jamais être missionnaire qu'animé d'une foi. L'efficacité des missionnaires chrétiens des $\mathrm{XIX}^{\mathrm{e}}$ et début du XX ${ }^{\mathrm{e}}$ siècle est liée à leur fondamentalisme (Fadipe 1970 : 288), qui croit au danger de mort éternelle qu'annonce le Christ, en même temps qu'à la bonne nouvelle du salut qui sauve de ce danger pour donner accès à la vie 
bienheureuse de l'éternité. Le type du missionnaire reste S. François Xavier, qui baptise à tour de bras dans l'urgence comme un pompier qui essaie d'éteindre un incendie, celui du feu de l'enfer engloutissant les païens.

31 Sous ce rapport, le message était le même qu'il s'agît de protestants ou de catholiques, tous alors éloignés du relativisme sceptique de notre fin de siècle. La "saga" catholique raconte à l'envi avec Mgr Hennemann (Skolaster 1924 : 239) que les néophytes posaient des charbons ardents sur la peau des hésitants pour leur montrer le mal qu'ils risquaient à refuser le baptême. En fait, tous prêchaient les vérités premières dans le même style ; ainsi le Dr Good, mort la veille de ses 38 ans chez les Bulu le 13 décembre 1894 (Fame Ndongo 1989 : 15 n.l), à peu près quand commence l'histoire de la mission catholique.

Selon le mémorial intitulé Nnanga Kon, ("le Fantôme Blanc" ; cf. L.T. 1981 : 170) voici son dialogue de conversion avec Angoneman, une jeune fille qu'il a arrachée à la pendaison lors du décès de son époux :

"L'âme a deux chemins : soit la mort dans un endroit où elle brûle pour toujours sans répit; soit la Vie dans un endroit ou règne éternellement la paix et où l'on ne connaît ni la maladie, ni la mort, ni le chagrin.

- (...) Où se trouve cet endroit? Comment est-il ?

- C'est Celui qui nous a tous créés et toutes les choses (...). Les âmes de ceux qui commettent le péché vont au mauvais endroit où sévissent continuellement les flammes. Tandis que celles des hommes qui posent des actes équitables vont dans l'endroit agréable pour y demeurer éternellement.

- Nous y irons donc tous parce que les gens d'ici ne font que du bien et des choses justes. Ils n'agissent que conformément aux recommandations de leurs fétiches (...).

Le missionnaire s'écrie qu'au contraire les fétiches qui voulaient tuer Angenoman ellemême, qui permettent magie, polygamie, razzia, esclavage, mauvais traitements aux femmes, mariage forcé, échange des épouses entre les maris, font commettre aux gens autant d'horribles péchés.

- Tous les gens pécheurs (...) iront dans le redoutable endroit où ils brûleront dans les flammes pour toujours.

- Heeg! Quel épouvantable endroit ! Je sais comme de petites brûlures au doigt font mal, combien plus passer toute sa vie dans le feu!

- Pas toute la vie ; mais éternellement. La vie d'ici bas est trop courte. Il ne faut pas comparer cette abominable situation avec l'éphémère vie humaine (...).

- Que faut-il faire pour échapper à ce feu ? (...).

- Ecouter attentivement! Quand Dieu... (...) vit que les hommes n'étaient pas justes, qu'ils ne le suivaient pas, il voulut les exterminer tous. Il eut encore pitié d'eux; il envoya dans ce monde son Fils afin qu'il leur montrât Dieu et les ramenât vers lui leur créateur (...).

- Comment s'appelait ce fils?

- Il s'appelle JESUS... Nous devions périr pour nos péchés, mais Dieu offrit Jésus pour qu'il mourût à notre place.

Le missionnaire se met alors à lire l'Evangile...

- Jésus dit aux pécheurs... Tu reconnais maintenant que tu es pécheresse?

- Très... lorsque tu as fini de me dire ce qu'est le péché, je me suis rendu compte que, si je meurs, mon âme ira certainement à ce lieu de feu (...). Parle ! Que dit Jésus aux pécheurs?

- Il dit : "Le sang de Jésus vous purifie de tout péché" ; "Venez à moi, vous tous qui êtes fatigués et chargés, je vous donnerais le repos". Et puis : "Je suis le chemin, je suis aussi la vérité et la vie ; nul ne vient à mon Père que par moi". Et aussi "Dieu a tellement aimé le monde qu'il a donné son Fils unique, afin que quiconque croit en lui ne périsse point, mais qu'il ait la vie éternelle". 

reçoit sur l'heure le baptême (Njemba Mendou 1932, traduit du bulu par Fame Ndongo 1989 : 101-105).

En voyage en 1901, le P. Vieter se désole d'être survenu trop tard pour baptiser un bébé d'un an qui vient de décéder : "Quel bel ange il eût fait au ciel !" (SVA 1901 : 154).

L'opposition des catholiques aux protestants est à chercher ailleurs. L'idée la plus précise en serait probablement fournie par l'étude du Premier Synode Camerounais de septembre 1906, où les supérieurs des postes missionnaires (Halbing, Nekes, Hoegn) se réunirent autour du Vicaire Apostolique Vieter, juste de retour de son sacre en Allemagne, pour instituer par décret les Statuts de la Mission catholique.

Ce long texte comporte certes des références évangéliques; mais l'essentiel en est constitué par des dispositions typiques du juridisme romain concernant l'organisation hiérarchique du Vicariat et les conditions de validité des sacrements. Ce dernier souci occupe 49 pages sur un total de 71.

L'élément qui surprend là le plus l'intellectuel relativiste, c'est que les décisions "obligatoires" (chap I art. V) du Synode entérinent une morale et une philosophie dites "naturelles", universelles, sur quoi se grefferait la religion. En particulier, en vertu de cette morale scolastique, le mariage est de soi monogame et indissoluble. Il en découle que le mariage païn est un véritable mariage. Le polygame qui se convertit devra déterminer parmi ses unions laquelle constitue ce mariage unique, par rapport auquel toutes les autres de ses relations jusque là considérées comme conjugales ne sont et n'auront jamais été que concubinages illégitimes. On voit quelles tensions dramatiques peuvent surgir : la question de savoir quelle épouse sera conservée comme la "vraie" constitue l'intrigue majeure du roman de Mongo Béti : Le Roi Miraculé.

De toutes ces prescriptions, retenons ici qu'il est enjoint de conférer le baptême dès qu'on le peut, en particulier aux païens en danger de mort.

41 Revenons-en à la perception initiale de cette entreprise par les Beti.

42 Ils n'en prennent connaissance qu'à partir de 1894, quand les premiers d'entre eux conduits par Dominik voient la mer à Kribi, nom qui, selon l'étymologie populaire (këlë mbil : "va au Trou" ; cf. L.T. 1985 : 49), signifie le bord du monde au-dessus du Gouffre de l'Océan.

43 Tout peut paraître exceptionnel en un tel lieu, avec un tel horizon. Un texte du premier converti et premier catéchiste beti, Martin Tabi (1911) permet de pressentir quelque chose de l'effet produit par la mission, école et surtout église, que les catholiques viennent d'y bâtir.

"Les Yaoundé lui donnaient le nom significatif de "Nda ngon Zamba", c'est-à-dire

de "maison de la fille de Dieu".

44

Ce nom est en effet fort expressif pour les Beti, car il rattache l'église à leur vieille mythologie. La "fille de Zamba" (cf. L.T. 1985 : 28-33) est une entité mystérieuse qui semble avoir eu un enfant incestueux de son père Zamba. Traduit à tort par "Dieu" (L.T. 1985 : 21-36), ou alors il faudrait dire "un dieu", le mot zamba parait désigner l'un des Invisibles suprêmes, Ancêtre moniteur et père des hommes. Quoi qu'il en soit, il a jeté ce fils au loin, et depuis sa fille le recherche sur terre en pleurant. On rendait à celle-ci un culte annuel de prémices au bord des rivières, car la fertilité des champs lui était attribuée; elle paraît être une sorte de souveraine des défunts, auxquels l'eau est 
associée, et à plus forte raison l'Océan assimilé à l'Endam, le fleuve des enfers qui entoure la terre et qui sépare les vivants des morts. construction en bois à trois nefs exécutée par des menuisiers d'Accra, "quoique très simple", constate son architecte, "cette église fit sur les Africains une impression profonde, car ils n'avaient jamais vu pareil édifice, de 26 mètres de long, 12 de large, 6 mètres 50 de haut, avec un petit clocher" où sont installées deux cloches (plus tard trois) dont la plus grosse était offerte par le village natal du P. Vieter, Kappenberg. A soi seul, l'édifice constituait un prodige.

"Les missionnaires, dans leurs habits ecclésiastiques, étaient pris pour les "Ngon Zamba", les filles de Dieu elles-mêmes. La description de l'authentique fille de Dieu par certains laissait sur sa faim: elle possédait quatre yeux, deux par-devant et deux par-derrière. Le soleil ornait son visage, et la lune sa nuque, etc...".

51 Logiquement, un temple mène à la manifestation du dieu qu'il renferme; dans mainte religion africaine, le prête est en certaines occasions, le dieu qu'il sert. L'évocation de la fille de Zamba semble être ici celle de la mythologie traditionnelle ; mais elle va bien plus loin. J'incline à croire qu'elle est très influencée par la vue, lors de la messe, des prêtres catholiques en ornements sacerdotaux, chapes et chasubles souvent brodées de signes cosmiques, de croix en gloire au milieu de soleils rayonnants de fils d'or ou d'argent etc. 
Vieter précise bien qu'il fait venir d'Allemagne les plus beaux vêtements possibles et tient à la splendeur traditionnelle du culte catholique, vantée par Goethe et les romantiques.

C'est sans aucun doute de cette vision qu'il faut partir pour reconstituer le processus de la croyance. Glissés parmi la foule lors d'une cérémonie de fête, les premiers témoins beti se demandent quels sont ces fantômes blancs chamarrés qui officient au son jusque là inouï des cloches, parmi des fleurs, entre des statues terrifiantes, sous des nuages d'encens et avec des chants exotiques étranges; quels sont ces voyants qui psalmodient et parlent avec autorité; ces dieux devant qui tous (même les autres Blancs présents) se prosternent.

53 La déduction s'impose : "Oui, c'est elle, c'est la déesse!" "Situés ainsi tout au bord du Gouffre, sous le signe des richesses et de la mort, devant un horizon d'une ampleur jamais vue, ces êtres ne peuvent être que des épiphanies de la redoutable fille de Zamba, dont l'église est la demeure (cf. SVA 1895 : 21, photo intérieure de l'église de Kribi). Mieux vaudra taire ce qu'on aura vu là.

Tabi prétend que, la première à Yaoundé, sa famille a nourri le projet de le mettre pensionnaire à la mission pour y apprendre la science des Blancs. Le fait n'est pas impossible : déjà certains Beti plus proches de la côte y envoyaient des enfants, tel le futur chef Max Abé Fouda de Nkolbewoa. Tabi dût attendre les fournées de Dominik, mais, dit-il,

"Je me mis à brûler du plus vif désir de me voir remplir ce plan inestimable (...).

La curiosité juvénile de contempler enfin la "nda ngon Zamba" dont j'avais si souvent entendu parler, la fière perspective de nouer bientôt une plus intime connaissance avec le kalara (livre) ne me laissaient plus de repos. Je dus patienter, on me trouvait trop jeune".

On retrouve ici l'envie de partir que nous trouvons exprimée par bien d'autres jeunes Beti de l'époque. Mais le désir de Tabi est mieux informé par une double orientation précise, religieuse et intellectuelle, dont les composantes sont, pour lui comme pour les missionnaires, les éléments indissociables d'une initiation globale. Après quelques péripéties, dont une émouvante séparation de sa mère, ce désir s'accomplira si bien que Tabi sera le principal artisan du transfert des "filles de Dieu" depuis le Gouffre jusqu'aux lointaines montagnes des Beti. On s'en tiendra là. Les Beti seront tous convertis en 30 ans. Mais 30 ans plus tard, à la suite $\mathrm{du} 2^{\text {ème }}$ concile $\mathrm{du}$ Vatican, leurs musiques et leurs danses à leur tour transformeront l'Eglise.

\section{BIBLIOGRAPHIE}

Abréviations : L.T. = LABURTHE-TOLRA

SVA = Stern von Afrika, (l'Etoile de l'Afrique) revue des PP. Pallotins, Limburg an der Lahn, 1894-1922. 
BERGER Heinrich, 1978 : "Die Katholische Mission in Kamerun während der Deutschen Kolonialzeit", Supplementa, Nouvelle Revue de Science Missionnaire, vol. XXVI, Verein zur Förderung der Missionswissenchaft, Immensee (Suisse), cartes, index, bibl. XXVII - 358 pp. in- $8^{\circ}$.

BOECKHELLER N. 1897, Theodor Christaller, der erste deutsche Reischs-schullehrer in Kamerun. Ein Lebensbild erzählt von... Illustrirte Familienbibliothek I, Buchhandlung für Innere Mission, Schw. Hall, Druck von Emil Hermann Senior Leipzig, 8 hors-texte, ill., 156 p. in-16.

CRIAUD Jean (C.S.Sp.) 1989, Ils ont planté l'Eglise au Cameroun : Les Pallotins, 1890-1915, Publications du Centenaire, Procure, B.P. 185 Yaoundé, 84 p., nombreuses photos.

CRIAUD J./VIETER 1990 : cf. VIETER.

DANSETTE Adrien 1965 : Histoire religieuse de la France contemporaine. L'Eglise catholique dans la mêlée politique et sociale, éd. revue et corrigée, collection "l'Histoire", Flammarion, 892 p.

FADIPE 1970 : The sociology of the Yoruba, edited \& with an introduction by F. Olu Okediji Ph. D., Ibadan University Press, 354 p. grand in- $8^{\circ}$.

FAME NDONGO 1989, cf. NJEMBA MEDOU.

LABURTHE-TOLRA P. 1981 : Les seigneurs de la forêt (Minlaaba I), essai sur le passé historique, l'organisation sociale et les normes éthiques des anciens Bëti du Cameroun, Publications de la Sorbonne, Paris, 490 pp. in- $8^{\circ}$.

LABURTHE-TOLRA P. 1985 : Initiations et sociétés secrètes au Cameroun : les Mystères de la Nuit (Minlaaba II) Karthala, Paris, 444 p.

LABURTHE-TOLRA P. 1991 : "Des fragments du ciel aux cultes du mal", in Les Fang, éditions du Musée Dapper, Paris.

MESSINA Jean-Paul 1988 : "Contribution des Camerounais à l'expansion de l'église catholique. Cas des populations du sud-Cameroun 1890-1961". Thèse présentée pour l'obtention du Doctorat en $3^{\circ}$ cycle d'histoire sous la direction du Prof. MVENG E., Université de Yaoundé, 390 pp. ronéo, index...

MOHR Hubert, 1965 : Katholische Orden und deutscher Imperialismus, mit einem Vorwort von M.M. Sejnman, Akademie-Verlag, Berlin (Ost), 360 p.

MONGO BETI, 1958 : Le roi miraculé, Buchet-Chastel, Paris, 256 p. in-8².

NJEMBA MEDOU Jean-Louis : 1932 (trad. 1989) : Nnanga Kon, premier roman écrit par un Camerounais, récit traduit, commenté, annoté par Jacques FAME NDONGO, maître de conférences à l'Université de Yaoundé, Sopecam, Yaoundé 1989, 158 p.

SKOLASTER H. 1924 : Die Pallotiner in Kamerun : 25 Jahre Missionsarbeit, Limburg-an-der Lahn, 327 p. in- $8^{\circ}$, tableaux.

Statuten der I. Synode in Kamerun abgehalten in Duala von 26 bis 28 September 1906 (publié 1907), $72 \mathrm{pp}$.

SUHAUD Charles 1976 : "Splendeur et misère d'un petit séminaire" in Actes de la Recherche en Sciences Sociales, Paris, $2^{\mathrm{e}}$ année, $\mathrm{n}^{\circ} 4$, pp. 66-90.

Synode cf. Statuten der...

TABI Isidore 1971 : "L'Eglise au Cameroun, 1884-1935", maîtrise d'histoire Faculté des Lettres et sciences humaines de Lyon, 152 I-III p. ronéo.

TABI Martin 1911 : (orthographié par erreur : SABI) : "Wie ich Christ wurde", revue Stern von Afrika, pp. 5-10. 
VIETER Heinrich 1890-1912, trad. et présentation de J. CRIAUD, Les premiers pas de l'Eglise au Cameroun, chronique de la mission catholique 1890-1912, récit de Mgr... Publications du Centenaire, Imprimerie St Paul, Yaoundé, 1989, 180 pp. in- $8^{\circ}$.

WEBER Max, 1903 : L'éthique protestante et l'esprit du capitalisme, suivi d'un autre essai, traduit de l'allemand par Jacques Chavy, Plon, Paris 1964, $2^{\text {e }}$ édition corrigée 1967, 342 p., avec références (chiffres entre parenthèses) à la collection Gesammelte Aufsätze zur Religions - Soziologie, Band I, 4 Aufl. Tübingen, reprenant l'édition de 1920-1903.

\section{RÉSUMÉS}

The intention of this article is to study variations in attitudes of mind. This can be especially observed in events, which may be qualified as odd, such as attempts at religious conversion, namely the coming about of a new way of thinking and the willingness to change forged in a society which up that point in time had lived in a different manner. Some events concerning the meeting of Catholic missionaries with the Beti of the southern Cameroons will be described. This unusual meeting led to an effective change in the way that both the Beti and the missionaries saw the world.

\section{AUTEUR}

\section{PHILIPPE LABURTHE-TOLRA}

Université René Descartes - Paris V - Sorbonne - Paris - France 